\title{
Parametric Study of Single-Walled Carbon Nanotubes Using Alcohol Catalytic Chemical Vapor Deposition
}

H. Emrah Unalan and Manish Chhowalla

Ceramic and Materials Engineering Department, Rutgers University

Piscataway, NJ 08854

\begin{abstract}
Single-walled carbon nanotubes (SWNTs) were synthesized both in powder form and on substrates using alcohol catalytic chemical vapor deposition method (ACCVD). A detailed parametric study was conducted. As-grown SWNTs were analyzed using transmission electron microscopy (HRTEM), field-emission scanning electron microscopy (FE-SEM) and Raman spectroscopy. Nucleation and growth of SWNTs occurred within seconds after introduction of the alcohol vapor. The results show that high quality SWNTs with a narrow diameter distribution without amorphous carbon can be grown using Co acetate catalyst doped with Fe acetate above $750^{\circ} \mathrm{C}$. Defective nanotubes were observed at low $\left(450^{\circ} \mathrm{C}\right)$ and high $\left(950^{\circ} \mathrm{C}\right)$ temperatures with the optimum temperature being $850^{\circ} \mathrm{C}$. In brief, our work has taken a step towards optimizing SWNTs growth conditions using ACCVD.
\end{abstract}

\section{INTRODUCTION}

The unique structural, mechanical, and electrical properties of single-wall carbon nanotubes (SWNTs) ${ }^{1}$ have rendered these structures highly appealing for investigators. The field of nanotube research is reaching maturity through demonstration of opto-electronic devices, field effect transistors, and sensors. One of the major obstacles to SWNT devices is the absence of control over the chirality during growth which determines their electronic and optical properties ${ }^{2}$.

Different methods have been employed for the synthesis of SWNTs such as alcohol catalytic chemical vapor deposition (ACCVD) ${ }^{3}$. However, no detailed parametric study of this method has been undertaken. In this work, we present a detailed analysis of the growth properties of SWNTs as a function of growth time, deposition temperature, alcohol flow rate, type of alcohol (i.e. ethanol versus methanol) and catalyst concentration (Fe:Co acetate ratio) to further emphasize their effect on the properties of SWNTs.

\section{EXPERIMENTAL DETAILS}

In the ACCVD method, alcohol vapor (either ethanol or methanol) is used as the carbon source and metal acetates (mixture of $\mathrm{Fe}$ and $\mathrm{Co}$-acetate) dissolved in water or alcohol were used as the catalyst materials. Heating the acetates to growth temperature $\left(450^{\circ} \mathrm{C}-950^{\circ} \mathrm{C}\right)$ leads to elimination of the organic layer, creating pure nano-sized catalyst particles which can be used for growth of SWNTs. The SWNTs were grown on a catalyst support bed (such as $\mathrm{MgO}$ ) and on substrates. Acetates were dissolved in solvent and solution was drop casted onto the $\mathrm{MgO}$ bed. The dip coating method was applied to substrates. The growth method is similar to the one described in Ref 4. except for the reduction step. $\mathrm{MgO}$ powder supported with catalyst particles were placed into alumina boats whereas a graphite stage was used to support the substrates. One inch quartz tube vacuum furnace was used for growth. The chamber was first evacuated to 150 
mTorr, and then the samples were heated up to the desired reaction temperature. The samples were kept at the growth temperature for 5 minutes in $250 \mathrm{sccm}$ of flowing argon. The argon was then shut off and the tube was evacuated before the introduction of alcohol vapor. The alcohol vapor is then transferred into the quartz tube to achieve a pressure of 5 to 10 Torr. After growth, the alcohol vapor was evacuated, argon was introduced and the tube is cooled to room temperature. The time of $\mathrm{MgO}$ growth was 20 minutes on $\mathrm{MgO}$ substrates and 50 minutes for substrate growth.

As grown samples were analyzed by Raman spectroscopy (Renishaw System 1000 Micro-Raman Spectrometer) with a laser wavelength of $785 \mathrm{~nm}$. SEM analysis was performed using Field Emission LEO Zeiss Gemini 982 Scanning Electron Microscope. The existence and morphology of the SWNTs were confirmed by Transmission Electron Microscopy (200 kV Ultra-high Resolution Transmission Electron Microscope TOPCON 002B).

SWNTs grown on $\mathrm{MgO}$ were ultrasonicated in methanol for TEM sample preparation, whereas SWNTs grown on quartz were transferred onto the grids by etching the top most layer of the quartz using $\mathrm{HF}: \mathrm{HNO}_{3}: \mathrm{H}_{2} \mathrm{O}(1: 1: 10)$ solution.

\section{RESULTS AND DISCUSSION}

We first conducted a temperature study. The furnace temperature was varied from $450^{\circ} \mathrm{C}$ to $950^{\circ} \mathrm{C}$ and samples were characterized using Raman spectroscopy monitoring the changes in radial breathing modes (RBMs), D-peak and G-peak. Raman spectra associated with furnace temperature study is given in the following figure. Figure 1a represents lower frequency region which contains RBM peaks while Figure 1 b shows D and G peaks which correspond to defects present in the carbon network and in-plane vibrations of a highly curved graphene sheet, respectively. For comparison, Raman spectrum is normalized with respect to G peak. Figure 2 shows SEM and TEM images of the as grown samples for three different furnace temperatures.
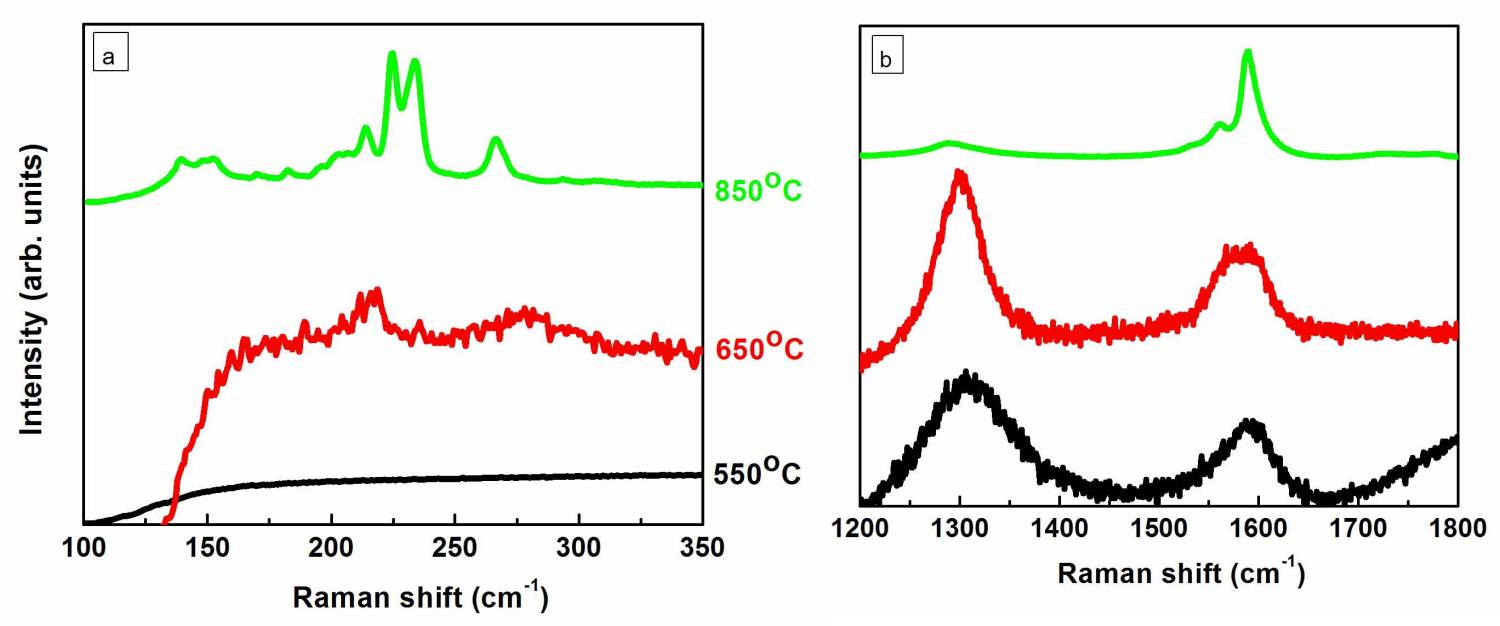

Figure 1. (a) Lower frequency and (b) higher frequency regions of the Raman spectra of nanotubes grown on $\mathrm{MgO}$ support material for different growth temperatures. 

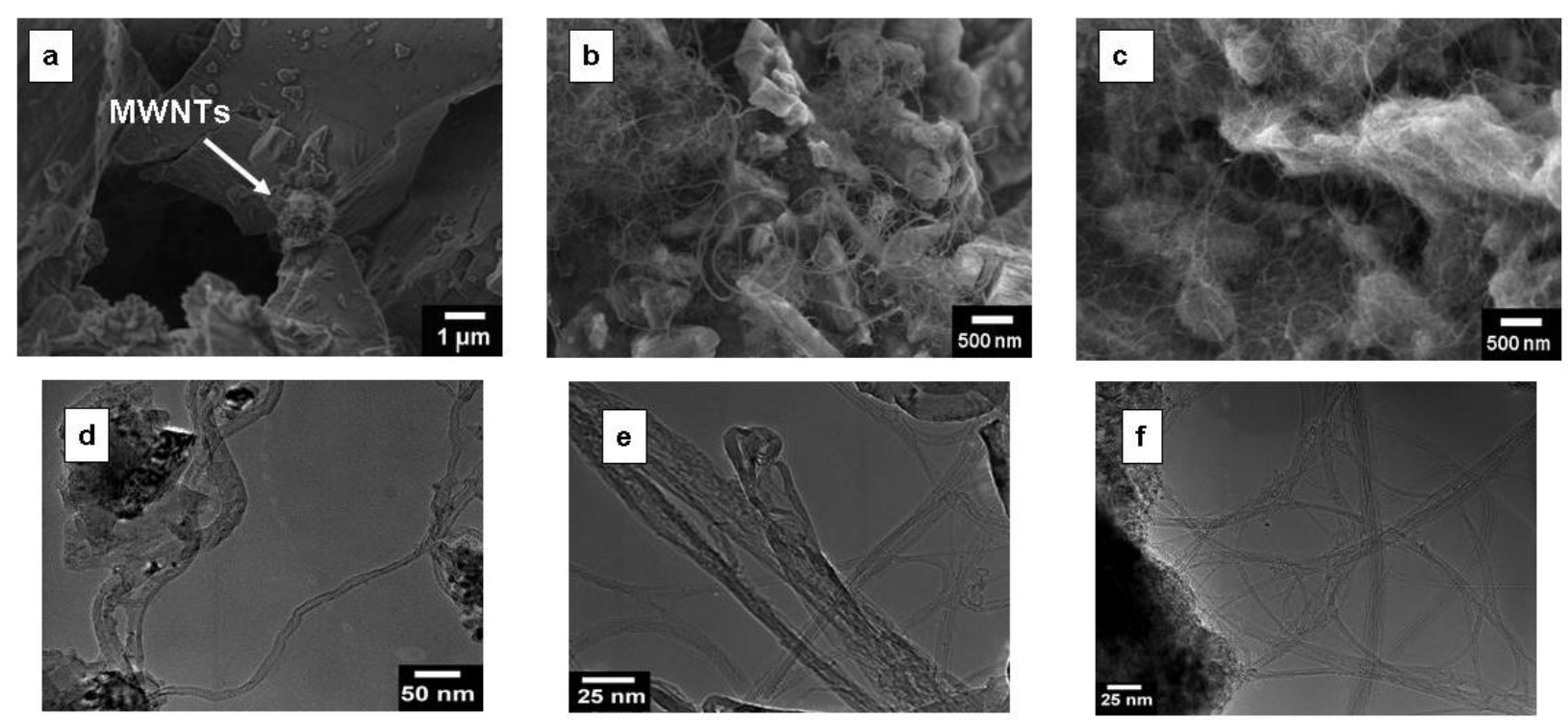

Figure 2. SEM images of as grown nanotubes corresponding to furnace temperatures (a) $450{ }^{\circ} \mathrm{C}$, (b) $650^{\circ} \mathrm{C}$ and (c) $850^{\circ} \mathrm{C}$.TEM images of as grown nanotubes corresponding to furnace temperatures (d) $450^{\circ} \mathrm{C}$, (e) $650^{\circ} \mathrm{C}$, (f) $850^{\circ} \mathrm{C}$.

It was found that nanotubes can be grown at temperatures as low as $450^{\circ} \mathrm{C}$ (Figure $2 \mathrm{a}$ ). Nanotubes grown at lower temperatures were multiwalled as seen in the TEM image shown in Figure $2 \mathrm{~d}$. The detection of RBM peaks at $650^{\circ} \mathrm{C}$ indicates that the nucleation of SWNTs begins near this temperature. Presence of SWNTs at $650^{\circ} \mathrm{C}$ was also confirmed with SEM (Figure 2b) and TEM (Figure 2e) observations. Nanotubes grown below $850^{\circ} \mathrm{C}$ were found to be a mixture of SWNTs and MWNTs. However, the fraction of SWNTs increases dramatically with temperature, reaching $100 \%$ at $850^{\circ} \mathrm{C}$ (Figure $2 \mathrm{c}, 2 \mathrm{f}$ ). Signatures of well-crystallized SWNTs, in other words strong presence of RBM modes, very small and broad D-peak and narrow and high intensity Gpeak with characteristic G-splitting was observed for the samples grown at $850^{\circ} \mathrm{C}$.

Intensity ratio of D-peak to the G-peak was used for monitoring the purity of SWNTs ${ }^{5}$. The magnitude of the $\mathrm{D}$ to G-peak ratio gradually decreased with the reaction temperature reaching its minimum value at $850^{\circ} \mathrm{C}$. The higher values of $\mathrm{D}$ peak, indicative of large quantities of amorphous carbon, can be attributed to non-optimized cracking of alcohol vapor at low temperatures.

A similar trend was observed for the samples grown on substrates. SEM and TEM images of nanotubes grown on quartz substrates are shown in Figure 3. 

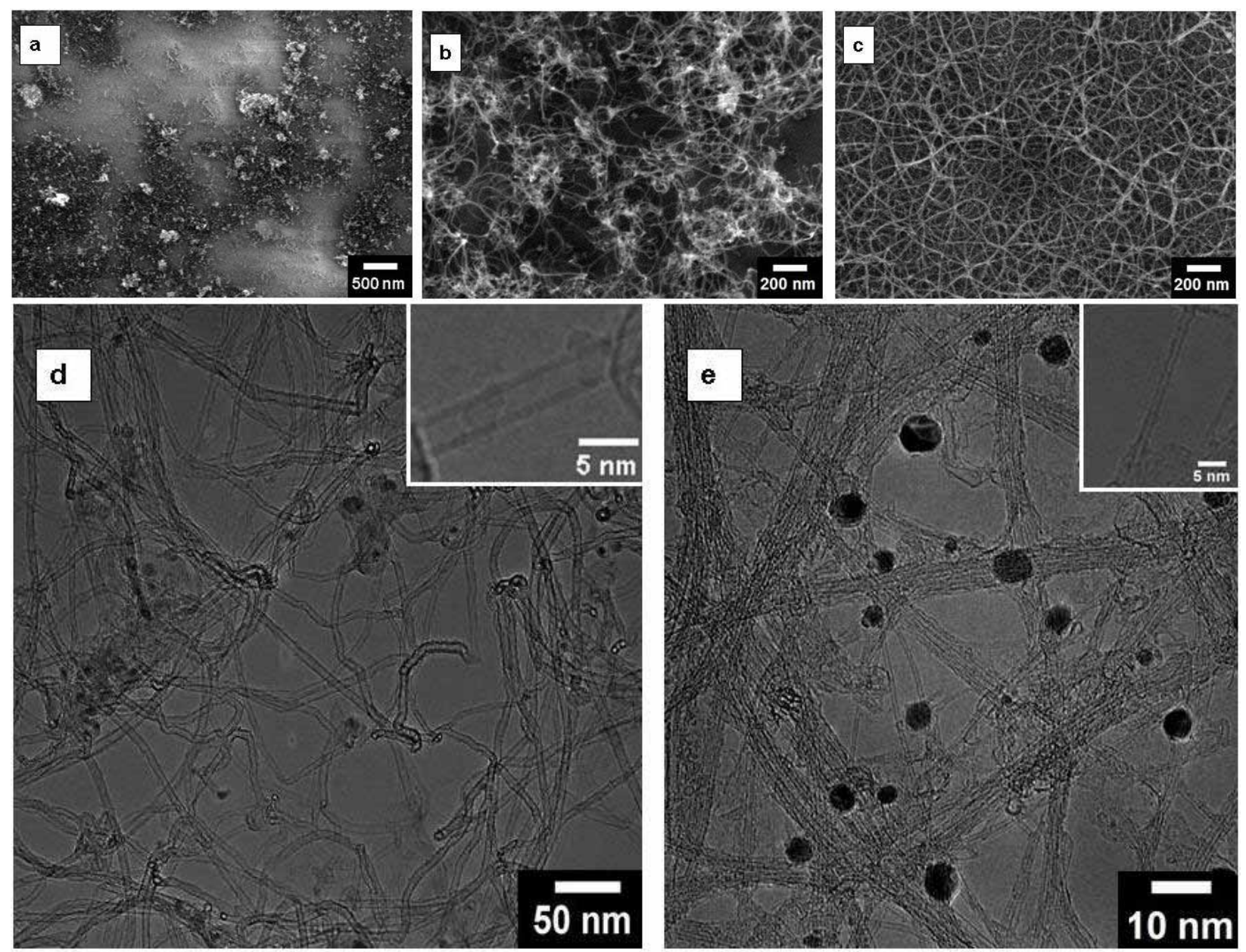

Figure 3. SEM images of as grown nanotubes with respect to furnace temperature (a) $450^{\circ} \mathrm{C}$, (b) $550^{\circ} \mathrm{C}$ and (c) $850^{\circ} \mathrm{C}$. TEM images of as grown nanotubes corresponding to furnace temperatures (d) $550^{\circ} \mathrm{C}$, (e) $850^{\circ} \mathrm{C}$.

For the samples grown on quartz, patchy growth was obtained at $450^{\circ} \mathrm{C} .2,3$ or 4 walled carbon nanotubes (Figure 3d) were present for the samples grown at $550{ }^{\circ} \mathrm{C}$. The threshold temperature for SWNT growth was obtained as $650^{\circ} \mathrm{C}$ from Raman spectroscopy (not shown here). Similar to the growth on $\mathrm{MgO}$, optimum growth temperature was determined to be $850^{\circ} \mathrm{C}$ for the nanotubes grown on substrates. Figure 3e shows TEM images of SWNTs grown at $850^{\circ} \mathrm{C}$, with the inset showing a single SWNT. Correlation between SWNT diameters and RBM frequencies was made using the empirical relationship ' $d=248 / \lambda{ }^{6}$, where $\lambda\left(\mathrm{cm}^{-1}\right)$ indicates the Raman shift and $d(\mathrm{~nm})$ indicates the nanotube diameter. The diameters of the SWNTs obtained from the radial breathing modes (RBM) are in good agreement with the diameters obtained from transmission electron microscope.

We then monitored the effect of alcohol flow on SWNT growth. The alcohol flow rate was controlled by a hot plate placed underneath the alcohol flask. Different hot plate temperature settings correspond to different alcohol flow rates. Dependence of alcohol flow rate as a function of the alcohol bath temperature is given in Figure 4. 


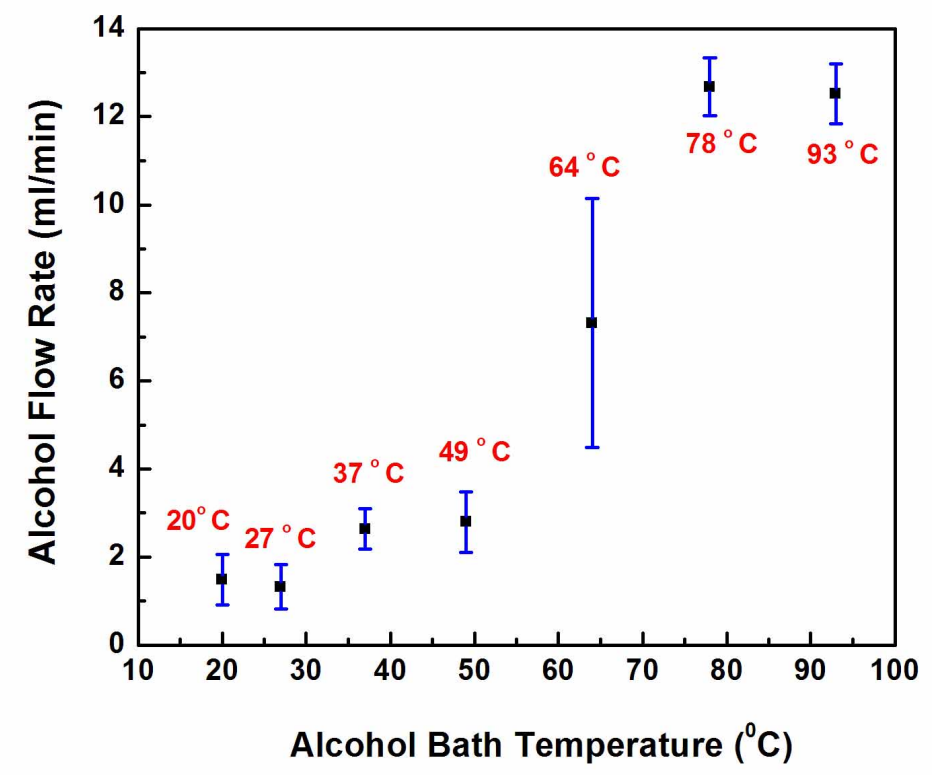

Figure 4. (a) Alcohol flow rate with respect to alcohol bath temperatures.

It can be seen in Figure 4 that higher alcohol bath temperatures correspond to higher alcohol flow rates. D over $\mathrm{G}$ peak ratios were found to be $0.06,0.14,0.25$ and 0.55 for alcohol bath temperatures of $27^{\circ} \mathrm{C}, 49^{\circ} \mathrm{C}, 64^{\circ} \mathrm{C}$ and $93^{\circ} \mathrm{C}$, respectively. However, it was observed from $\mathrm{D} / \mathrm{G}$ ratios that intensity of D-peak increases with alcohol flow. In other words, deposition of unwanted amorphous carbon takes place at higher alcohol flow rates, and increases continuously with alcohol flow.

The last parameter investigated was the solvent used to dissolve the catalyst particles. Three different solvents were utilized, namely ethanol, methanol and deionized water (DI). After ultrasonication for 2 hours, no precipitation was observed for any of the three solvents. The substrates were then dip coated with those solutions. The tension balance for a liquid drop on a substrate surface is shown in Figure 5.

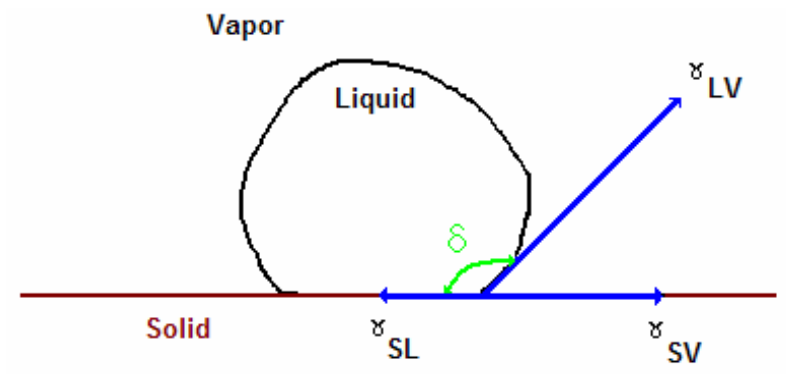

Figure 5. The surface tension force balance for a liquid drop on a surface.

Surface tension equation can be written as $\gamma_{\mathrm{SV}}=\gamma_{\mathrm{SL}}+\gamma_{\mathrm{LV}} \times \cos \delta$, where $\gamma$ stands for surface tension and $\delta$ is called as dihedral angle. Complete wetting occurs when the drop spreads freely over the solid, which corresponds to the equation: $\gamma_{\mathrm{SL}} \leq \gamma_{\mathrm{SV}}-\gamma_{\mathrm{LV}}$, where $\mathrm{S}, \mathrm{L}$ and V represent substrate, catalyst solution and air respectively in our system. Therefore, the modified surface tension balance is: $\gamma_{\text {Quartz-Solvent }} \leq \gamma_{\text {Quartz-Air }}-\gamma_{\text {Solvent-Air }}$. The surface tension of the water is higher compared to the alcohols due to its strong hydrogen bonds. Hence, the wetting of the 
water which carries catalyst particles on the substrate surface is better than the alcohols. This means that there is uniform catalyst distribution. Consequently, homogeneous nanotube growth over the entire substrate occurs when catalyst particles are dissolved in DI water. Non-uniform growth of nanotubes using catalyst particles dissolved in ethanol and methanol is shown in Figures $6 \mathrm{a}$ and $\mathrm{b}$.

The higher frequency Raman features for the samples grown with the solvents were identical since all three samples were grown with the same process parameters. Lower frequency RBM features attributed to diameter distribution of the SWNTs were similar since no precipitation was observed in either of the catalyst solutions.
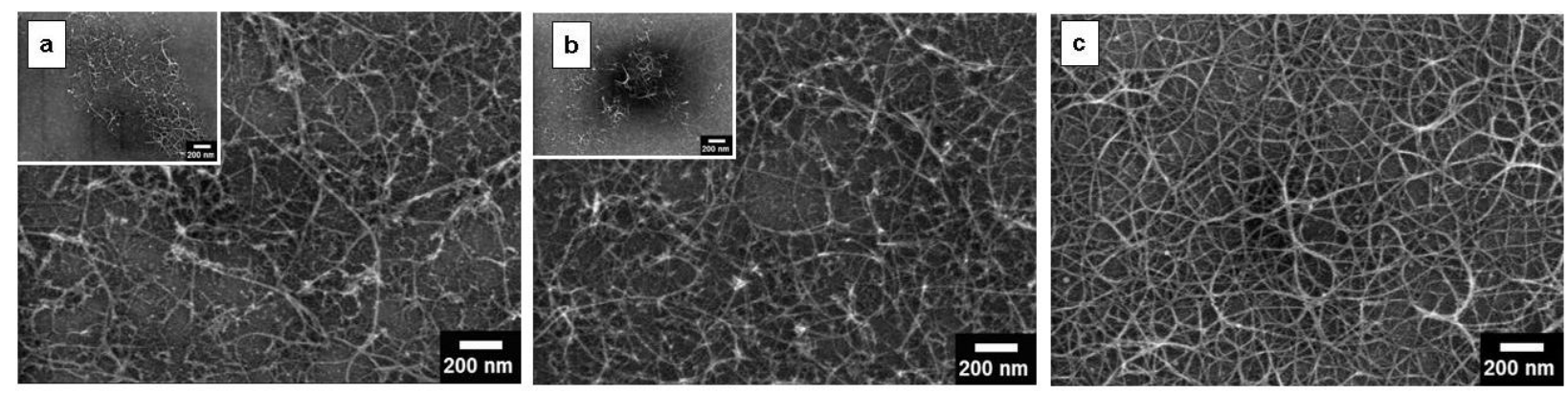

Figure 6. SEM images for nanotubes grown with the catalyst particles dissolved in (a) methanol, (b) ethanol and (c) deionized water. Inset shows the scarce areas with nanotubes. Associated Raman spectra for SWNTS grown with all three the solvents (d) high (e) low frequency region.

\section{CONCLUSION}

In conclusion, SWNTs were grown using alcohol catalytic method. $850^{\circ} \mathrm{C}$ is found to be the optimum growth temperature. There is enough alcohol volatilization at room temperature for SWNT growth which leads to amorphous carbon free growth. Deionized water was used as an efficient solvent for catalyst particles so that uniform growth of SWNTs can take place over the entire substrate surface.

\section{ACKNOWLEDGEMENTS}

This work is supported by a NSF-ECS Grant 0400501.

\section{REFERENCES}

[1] S. Iijima, T.Ichihashi, Nature 363 (1993) 603

[2] M.S. Dresselhaus, G.Dresselhaus, P.Avouris, Carbon Nanotubes, Sringer, Berlin, 2001.

[3] S. Maruyama, R.Kojima, Y.Miyauchi, S. Chiashi, M. Kohno, Chemical Physics Letters 360 (2002) 229

[4] Y.Murakami, S.Chiashi, Y.Miyauchi, M.Hu, M.Ogura, T. Okubo, S. Maruyama, Chemical Physics Letters 385 (2004) 298

[5] H. Kataura, Y. Kumazawa, Y.Maniwam Y.Otsuka, R.Sen, S.Suzuki, Y.Achiba, Carbon 38 (2000) 1691

[6] R.Saito, G.Dresselhaus, M.S.Dresselhaus, Phys. Rev. B 61 (2000) 2981 